\title{
Bioenergetic Signature in Non-Muscle Invasive Urinary Bladder Cancer Progression: low grade $\mathrm{pTa}$, high grade $\mathrm{pTa}$, $\mathrm{pT} 1$
}

\section{Arthur D. Ottaiano*, Gabriel C. S. Simões, Guilherme A. P. Costa, Wagner J. Fávaro, Petra K. Böckelmann}

\begin{abstract}
The urinary bladder carcinoma $(\mathrm{BC})$ is the second most common malignant disease of the urinary tract. The uncontrolled cell proliferation of these tumors is one of the main caracteristics of the neoplastic cell. The goal of this study is to characterize and compare profiles of cellular energy metabolism on the different histological grades of NMIBC to, if possible, establish a Clinical-pathological and prognostic relevance criteria to classify those tumors.
\end{abstract}

\section{Key words:}

Bladder cancer, metabolism, immunohistochemical.

\section{Introduction}

The urinary bladder carcinoma $(\mathrm{BC})$ is the second most common malignant disease of the urinary tract and it is estimated, in the USA, that there will be about 79.030 new cases of bladder cancer in 2017 and 16.870 deaths will occur due this disease (1). More than $70 \%$ of the BC incidence is superficial (pTis, pTa and pT1), non-muscle invasive tumor (NMIBC) (2). However, $50 \%$ of the nonmuscle invasive tumors resort in 4 years after the treatment and $11 \%$ evolve to the invasive Phenotype (3). The uncontrolled cell proliferation of these tumors is one of the main characteristics of the neoplastic cell. For such thing, energy metabolism adjustments are necessary to stimulate the growth and division of the cell. Thus, the general goals of this study are to characterize and compare profiles of cellular energy metabolism on the different histological grades of NMIBC to, if possible, establish Clinical-pathological and prognostic relevance criteria to classify those tumors.

\section{Results and Discussion}

20 samples of urinary bladder tissue from men between the ages of 60 and 90 with and without urothelial lesions diagnosis, obtained in the Urology Service from the Amaral Carvalho Hospital (Jaú-SP) were used in this study. The patients were divided in 4 groups ( 5 samples each): Control group (no lesion); pTa low grade NonMuscle Invasive Group; pTa high grade Non-Muscle Invasive Group and pT1 Non-Muscle Invasive Group. Then the samples were submitted to histopathological analyzes to confirm the diagnosis, and after that, immunohistochemical of the GLUT 1 and LDH antigens was performed. The control and low-grade pTa groups have shown an intense immunolabeling of GLUT1 transporter, while high-grade pTa and pT1 groups have shown a moderate immunolabeling. This difference is related to the neoplastic lesion aggressiveness, that is, there is a increase of the oxygen and glucose need, witch exceeds the supply capacity of the vascularization, leading to the decrease of GLUT1 levels. The pTa groups neoplastic cells, according as they increase their malignancy, also increases their nutrients and oxygen deprivation, converting most of pyruvate that comes from glucose $(>90 \%)$ into lactate by the Lactate dehydrogenase (LDH), increasing these enzyme level. In pT1 group, since the neoplastic cells already invaded the stroma, thereafter meet with the blood vessels that are in there, besides the stimulus and formation of new vessels, what increases nutrients and oxygen levels for these cells, Decreasing the conversion of pyruvate to lactate by $\mathrm{LDH}$ and consequently decreasing the levels of this protein.

Conclusions
Due to all considerations above, our
immunohistochemical results show a discreet but present
metabolic behavior difference between the studied
groups.

\section{Acknowledgement}

Support by FAPESP-Brazil (Process numbers 2015/22799-6; 2014/11866-1).

\footnotetext{
References:

1 Altekruse et al., 2010; Zhang et al., 2011; Siegel et al., 2012; American Cancer Society, 2017

${ }^{2}$ Montironi \& Lopez-Beltran, 2005; Zhang et al., 2011; Askeland et al., 2012

${ }^{3}$ Shimada et al., 2011; Zhang et al., 2011; Askeland et al., 2012
} 